\title{
ALCANCES DEL NUEVO SISTEMA "TIPO PARA LA EVALUACIÓN DEL DESEMPEÑO LABORAL" (ACUERDO 565 DE 2016), DE LOS SERVIDORES PÚBLICOS DE CARRERA ADMINISTRATIVA, EN EL MARCO DEL DESARROLLO ORGANIZACIONAL ${ }^{1}$
}

$\underline{\text { Humberto Luís García Cárcamo }}^{2}$

Fecha de recepción: 16 de noviembre de 2016

Fecha de aceptación: 13 de diciembre de 2016

SUMARIO: 1. Introducción; 2. Marco normativo; 3. Nuevo Sistema "Tipo de evaluación del desempeño laboral" (Acuerdo 565 de 2016); 4. Motivos por los cuales la CNSC estableció un nuevo sistema "Tipo de evaluación del desempeño laboral" (Acuerdo 565 de 2016), para los servidores públicos de carrera, en el marco del desarrollo organizacional; 5. Diferencias entre el nuevo sistema (Acuerdo 565 de 2016) y el anterior sistema (Acuerdo 137 de 2010); 6.

Conclusiones; 7. Referencias bibliográficas.

1 Este producto de investigación fue fruto de una investigación realizada en el desarrollo de la Especialización en Alta Gerencia Universidad militar Nueva Granada, con recursos propios.

2 Abogado, especialista en Alta Gerencia Universidad militar Nueva Granada. 


\section{COMO CITAR ESTE ARTÍCULO (APA 6)}

García Cárcamo, Humberto Luís (2017). Alcances del nuevo sistema "tipo para la evaluación del desempeño laboral" (Acuerdo 565 de 2016), de los servidores públicos de carrera administrativa, en el marco del desarrollo organizacional. Revista Jurídica Mario Alario D’Filippo, IX (17), pág. 33-42.

\section{RESUMEN}

Dadas las repercusiones que implica la Evaluación del Desempeño Laboral tanto en el Estado Colombiano como en los servidores públicos y en últimas, en la ciudadanía en general, resulta de vital importancia analizar cuáles son los alcances del nuevo Sistema "Tipo de Evaluación del Desempeño Laboral" en el marco del desarrollo organizacional expedido por la Comisión Nacional del Servicio Civil a través del Acuerdo 565 de 2016 que responda a los principios de objetividad, igualdad y mérito. Lo anterior, en razón a que la Evaluación del desempeño laboral no sólo permite el acceso de los servidores públicos a la carrera administrativa en Colombia sino también, su permanencia en la misma, a través de estándares de calidad, trabajo en equipo y trabajo individual que finalmente, debe conllevar a la consecución del cumplimiento de las metas institucionales, planes de acción y planes operativo anual. En consecuencia, a través del siguiente trabajo, se establecerán los alcances del nuevo sistema "Tipo de Evaluación del Desempeño Laboral", para los servidores públicos de carrera, en el marco del desarrollo organizacional. Los motivos por los cuales la CNSC estableció un nuevo sistema "Tipo de Evaluación del Desempeño Laboraly las principales diferencias entre el nuevo sistema "Tipo de Evaluación del Desempeño Laboral” y el anterior Sistema "Tipo de Evaluación del Desempeño Laboral".

\section{PALABRAS CLAVES}

Evaluación laboral, desempeño laboral, merito, carrera administrativa, desarrollo organizacional.

\section{ABSTRACT}

Given the repercussions of the Labor Performance Assessment in both the Colombian State and public servants, and ultimately in the general public, it is vitally important to analyze the scope of the new "Type of Evaluation of Work Performance" Within the framework of organizational development issued by the National Civil Service Commission through Agreement 565 of 2016 that responds to the principles of objectivity, equality and merit. This is due to the fact that the Labor Performance Evaluation not only allows public servants access to the administrative career in Colombia but also their permanence in the same, through standards of quality, teamwork and individual work that Finally, must lead to the achievement of the institutional goals, action plans and annual operational plans. Consequently, through the following work, the scope of the new "Type of Evaluation of Work Performance" system will be established for career civil servants, within the framework of organizational development. The reasons why the CNSC established a new system "Type of Evaluation of Work Performance and the main differences between the new system" Type of Evaluation of Work Performance "and the previous" Type of Evaluation of Work Performance "system.

\section{KEYWORDS}

Job evaluation, job performance, merit, administrative career, organizational development. 


\section{INTRODUCCIÓN}

La evaluación del desempeño laboral en el sector público en Colombia, empezó a ser señalada en el Decreto 2400 de 1968 expedido por el Gobierno Nacional, el cual en los articulos 15, 16 y 17 establecieron que el rendimiento, calidad del trabajo y el comportamiento del empleado, sería objeto de calificación periódica y que una de sus ventajas era para la concesión de estimulos de carácter moral o pecuniario. También indicaba la obligatoriedad de "calificar los servicios" por parte del evaluador.

Luego, el Decreto 1950 de 1973, expedido por el Gobierno Nacional, en los articulos 228 a 238, se refiere a la evaluación del desempeño laboral como "calificación de Servicios". Establece también que los alcances de la "Calificación de Servicios" es para determinar la permanencia o el retiro del servicio, participar en los concursos para ascensos, formular programas de adiestramiento o perfeccionamiento, evaluar los sistemas de selección de personal e ingreso al servicio, otorgar becas o comisiones de estudio y conceder estimulos a los empleados y determinar la prioridad para la participación en los programas de bienestarsocial.

El Decreto 770 de 1988, derogó los articulos 228 a 238 del Decreto 1950 de 1973, relacionado con la "calificación de servicios" de los servidores públicos con derechos de carrera administrativa y en su lugar estableció unas nuevas directrices sobre esta materia, en especial, los recursos que proceden, los principios que la rigen, los mecanismos de notificación, los periodos que se tienen en cuenta para efectuar la calificación, el rol de emitir los formatos de calificación por parte del Departamento Administrativo de la Función Pública y sus alcances.

La Constitución Política de 1991, señala en su articulo 125 de 1991, señala que el ingreso a los cargos de carrera y el ascenso en los mismos, se harán previo cumplimiento de los requisitos y condiciones que fije la ley para determinar los méritos y calidades de los aspirantes y que el retiro será como consecuencia de la calificación no satisfactoria en el desempeño del empleo; por violación del régimen disciplinario y por las demás causales previstas en la Constitución o la ley.

De otra parte, si hay un "incumplimiento" en el contexto de una evaluación del desempeño del servidor con derechos de carrera que conlleva a la imposibilidad de alcanzar las metas y objetivos concretos definidos para cada cargo y función a través de una evaluación del desempeño no satisfactoria, implica el retiro del funcionario de la entidad en la cual presta sus servicios. (Corte Constitucional, 2005)

La Ley 909 de 2004, señaló en sus articulos 37 a 43, la "Evaluación del Desempeño Laboral", los principios que la orientan, los instrumentos de evaluación, qué implica para el servidor con derechos de carrera el resultado de la "Evaluación del Desempeño Laboral”, las consecuencias de su incumplimiento, la obligación de evaluar, los recursos 
que contra ella procede y quienes son los encargados de evaluar.

Finalmente, en virtud de la competencia asignada por la Ley 909 de 2004 a la CNSC, para reglamentar la Evaluación del Desempeño Laboral de los Servidores Públicos en Colombia, expidió el Acuerdo 017 de 2008, el cual fue derogado por el Acuerdo 137 de 2010 y en la actualidad, el Acuerdo 565 de 2016, cuya vigencia inició a partir del 1 de febrero del año 2017 y que derogó el Acuerdo 137 de 2010.

\section{MARCO NORMATIVO}

Para comprender jurídicamente la concatenación del acuerdo con el sistema normativo es necesario precisar su marco normativo, el cual consideramos que es el siguiente:

\begin{tabular}{|c|c|}
\hline $\begin{array}{l}\text { Constitución Política Colombia de } \\
1991\end{array}$ & $\begin{array}{l}\text { El articulo } 125 \text {, Señala que un servidor público con derechos de } \\
\text { carrera podrá ser retirado del servicio por evaluación del } \\
\text { desempeño no satisfactoria. }\end{array}$ \\
\hline $\begin{array}{l}\text { Ley } 909 \text { de 2004:"por la cual se } \\
\text { expiden normas que regulan el } \\
\text { empleo público, la carrera } \\
\text { administrativa, gerencia pública y se } \\
\text { dictan otras disposiciones". }\end{array}$ & $\begin{array}{l}\text { Los articulos } 38 \text { y } 41 \text { Señalan que la evaluación del desempeño se } \\
\text { efectuará bajo los parámetros previamente establecidos por la } \\
\text { CNSC, su definición, y que un resultado no satisfactorio en el } \\
\text { proceso de evaluación del desempeño laboral conlleva al retiro del } \\
\text { servicio por declaratoria de insubsistencia. }\end{array}$ \\
\hline $\begin{array}{l}\text { Decreto } 2539 \text { de 2005: "Por el cual se } \\
\text { establecen las competencias } \\
\text { laborales generales para los empleos } \\
\text { públicos de los distintos niveles } \\
\text { jerárquicos de las entidades a las } \\
\text { cuales se aplican los Decretos-ley } 770 \\
\text { y } 785 \text { de 2005". }\end{array}$ & $\begin{array}{l}\text { El articulo } 2^{\circ} \text { establece el concepto de lo que es una competencia } \\
\text { laboral, indicando que es la capacidad de una persona para } \\
\text { desempeñar en diferentes contextos y con criterios de calidad las } \\
\text { funciones del empleo a desempeñar }\end{array}$ \\
\hline $\begin{array}{l}\text { Circular No. } 04 \text { de 2005: “Evaluación } \\
\text { institucional por dependencias en } \\
\text { cumplimiento de la ley } 909 \text { de } 2004 .\end{array}$ & $\begin{array}{l}\text { Señala el procedimiento que debe hacer el jefe de control interno } \\
\text { en las entidades para realizar la evaluación de gestión por áreas o } \\
\text { dependencias y que genera una calificación para los servidores con } \\
\text { derechos de carrera. }\end{array}$ \\
\hline $\begin{array}{l}\text { Decreto } 1227 \text { de 2005: "por el cual se } \\
\text { reglamenta parcialmente la Ley } 909 \\
\text { de } 2004 \text { y el Decreto-ley } 1567 \text { de } \\
1998 \text { ". }\end{array}$ & $\begin{array}{l}\text { El articulo 50, } 51 \text { y } 52 \text { define las obligación de evaluar, por parte } \\
\text { del superior jerárquico del evaluado, los recursos contra la } \\
\text { calificación anual u ordinaria, el cual será presentado mediante } \\
\text { Recurso de Reposición ante el evaluador y Recurso de Apelación } \\
\text { ante el superior jerárquico del evaluado y las fases del proceso de } \\
\text { evaluación del desempeño laboral. }\end{array}$ \\
\hline
\end{tabular}


Establece todo el proceso de evaluación del Desempeño Laboral, Acuerdo 565 de 2016: "Por el cual añadiendo que será materia de evaluación no sólo los compromisos se establece el Sistema Tipo de laborales como tradicionalmente se hacía, sino también las Evaluación del Desempeño Laboral competencias comportamentales de tipo individual y la evaluación de los empleados públicos en carrera de gestión por áreas o dependencias, que será de tipo grupal. administrativa $y$ en periodo de prueba".

Señala que el periodo de evaluación de desempeño laboral que es del 1 de febrero al 31 de enero de la siguiente vigencia.

\section{NUEVO SISTEMA “TIPO DE EVALUACIÓN DEL DESEMPEÑO LABORAL” (ACUERDO 565 DE 2016)}

El servidor público con derechos de carrera no sólo será evaluado de manera individual a través del cumplimiento de los compromisos laborales, sino que también, le será evaluado, las competencias comportamentales que deberá demostrar en todo el proceso de evaluación del desempeño y adicionalmente, será motivo de calificación la evaluación de la gestión por áreas o dependencias, que se traduce en el trabajo en equipo. Es decir, que bajo esta óptica, no sólo deberá demostrar unas habilidades y competencias de tipo individual sino también de tipo grupal.

Es así como la Comisión Nacional del Servicio Civil, a través de la expedición del Acuerdo 565 de 2016, en su articulo 16 estableció unos componentes de evaluación de la siguiente forma:

\begin{tabular}{|l|l|}
\hline \multicolumn{1}{|c|}{ CALIFICACIÓN PARA EL PERIODO ANUAL U ORDINARIA } & \multicolumn{1}{c|}{ PESO PORCENTUAL } \\
\hline Compromisos laborales & $80 \%$ \\
\hline Competencias Comportamentales & $10 \%$ \\
\hline Evaluación de Gestión por Áreas o Dependencias & $10 \%$ \\
\hline Total & $100 \%$ \\
\hline
\end{tabular}

El desarrollo de las competencias y habilidades del servidor con derechos de carrera administrativa, también es tenido en cuenta en el sector privado por los directivos, los cuales también pueden evaluarlas, teniendo como bases la visión y misión de la empresa y alcanzar de esta forma los objetivos de la organización. Estas competencias pueden ser la orientación a resultados, el trabajo en equipo, la iniciativa, la integridad, la orientación al cliente y el empoderamiento.(Capuano, 2004)

Es así como resulta determinante las competencias laborales que son definidas como el conjunto de conocimiento, habilidades y actitudes que aplicadas o demostradas en situaciones del ámbito productivo, tanto en un empleo, como en una unidad para la generación de ingreso por cuenta propia, se traducen en resultados efectivos que contribuyen al logros de los objetivos por parte de la organización(Castillo, 2010)

Téngase en cuenta que una evaluación del desempeño integral es aquella en la cual el 
servidor público genera valor agregado a la entidad a través del desempeño efectivo y el cumplimiento de los compromisos laborales, competencias comportamentales, complementado con un trabajo en equipo(ESAP, 2012)

Finalmente, se puede afirmar que la evaluación de Gestión por áreas o dependencias, se basa en la evaluación cualitativa del grado de ejecución de los compromisos establecidos en los planes de desarrollo, planes de acción y planes estratégicos de la entidad, a través de los servidores que hacen parte de la entidad y su nivel de cumplimiento de los mismos. (bogota, 2015)

Observación: Se entrega en físico CD que contiene la entrevista efectuada el 23 de marzo de 2017, a la Directora de Administración de la Carrera Administrativa de la Comisión Nacional del Servicio Civil - CNSC- Dra. Sonia Patricia Cruz Ortega, quien tiene a su cargo la organización, reglamentación y expedición de las directrices a nivel nacional en materia de Evaluación del Desempeño Laboral y a quien se le preguntó ¿ cuáles fueron los alcances del nuevo "Sistema Tipo de Evaluación del Desempeño Laboral" Acuerdo 565 de 2016 ? y las motivaciones que conllevaron a que la CNSC expidiera dicho Acuerdo.

\section{MOTIVOS POR LOS CUALES LA CNSC ESTABLECIÓ UN NUEVO SISTEMA "TIPO DE EVALUACIÓN DEL DESEMPEÑO LABORAL" (ACUERDO 565 DE 2016), PARA LOS SERVIDORESPÚBLICOS DE CARRERA, ENEL MARCO DELDESARROLLOORGANIZACIONAL}

La CNSC al momento de reglamentar el nuevo sistema "Tipo de Evaluación del Desempeño Laboral" Acuerdo 565 de 2016, tuvo en cuenta que para que pudiera ser considerado una verdadera herramienta de gestión al interior de la entidad, debía involucrar no solo los compromisos laborales del servidor con derechos de carrera administrativa, sino también sus habilidades a través de la calificación de las competencias comportamentales y el trabajo en equipo a través de la evaluación de gestión por áreas o dependencias.

Es así como en las consideraciones establecidas en el Acuerdo 565 de 2016, la CNSC indicó:

Que mediante Acuerdo No. 137 del 14 de enero de 2010, se estableció el sistema tipo de Evaluación del Desempeño Laboral de los empleados de Carrera Administrativa y en período de prueba. No obstante, luego de su aplicación y conforme al estudio técnico realizado, se ha identificado la necesidad de actualizar dicha reglamentación, con el fin que la evaluación del Desempeño Laboral sea una herramienta de Gestión del Talento Humano (CNSC, Acuerdo 565, 2016).

La Evaluación del Desempeño podría convertirse en una potencial y eficaz herramienta de gestión para los servidores públicos con derechos de carrera si se pudiera concatenar con una evaluación de gestión por áreas o dependencias con resultados favorables para el mejoramiento institucional (Rodríguez, 2013), dado que aunando esfuerzos por parte de los servidores públicos en la consecución de una meta para su área, se obtendrían mejores resultados. 
De otra parte, dentro de las consideraciones para que la CNSC estableciera un nuevo sistema "Tipo de Evaluación del Desempeño Laboral", encontró que bajo las directrices del anterior Acuerdo 137 de 2010, los servidores públicos con derechos de carrera administrativa a los cuales solo les calificaban de manera individual, su desempeño podía resultar en el nivel sobresaliente, sin embargo, la evaluación del desempeño de la entidad, arrojaba un resultado no satisfactorio. (CNSC, Comisión Nacional del servicio Civil, 2016). Por tal motivo, la forma de poder mejorar este desempeño de la entidad, era a través de la implementación de la evaluación de gestión por áreas o dependencias mediante el trabajo en equipo de los servidores con derechos de carrera, mejorando de esta forma la prestación del servicio y por ende, una real correspondencia entre el desempeño individual y el desempeño institucional.

Finalmente, el servidor con derechos de carrera administrativa, no sólo debe demostrar que ha cumplido eficazmente con sus compromisos laborales sino que adicionalmente, debe cumplir con unas competencias comportamentales para la obtención de los objetivos y resultados esperados por parte de la entidad y que son definidas como:

Habilidades y conductas que muestran desempeños superiores en el trabajo y que generalmente se verbalizan en términos de atributos o rasgos personales, como es el caso de la orientación al logro, la pro actividad y el liderazgo. (Garcia,2014).

Observación: Se entrega en físico CD que contiene la entrevista efectuada el 23 de marzo de 2017, a la Directora de Administración de la Carrera Administrativa de la Comisión Nacional del Servicio Civil - CNSC- Dra. Sonia Patricia Cruz Ortega, quien tiene a su cargo la organización, reglamentación y expedición de las directrices a nivel nacional en materia de Evaluación del Desempeño Laboral y a quien se le preguntó ¿cuáles fueron los alcances del nuevo "SistemaTipo de Evaluación del Desempeño Laboral" Acuerdo 565 de 2016? y las motivaciones que conllevaron a que la CNSC expidiera dicho Acuerdo.

\section{DIFERENCIAS ENTRE EL NUEVO SISTEMA (ACUERDO 565 DE 2016) Y EL ANTERIOR SISTEMA (ACUERDO 137 DE 2010)}

Al verificar el contenido del Acuerdo 137 de 2010 y del Acuerdo 565 de 2016, se encuentran las siguientes diferencias:

- En cuanto a la calificación de evaluación de Gestión por áreas o dependencias, en el Acuerdo 137 de 2010, era uno de los factores cualitativos para acceder al nivel sobresaliente, mientras que en el Acuerdo 565 de 2016, tiene un peso porcentual del $10 \%$ sobre el $100 \%$ de la evaluación del desempeño laboral del servidor con derechos de carrera administrativa. 
- En lo pertinente a la calificación de las competencias comportamentales, en el Acuerdo 137 de 2010, era uno de los factores cualitativos para acceder al nivel sobresaliente, mientras que en el Acuerdo 565 de 2016, tiene un peso porcentual del $10 \%$ sobre el $100 \%$ de la evaluación del desempeño laboral del servidor con derechos de carrera administrativa.

- Los compromisos laborales, en el Acuerdo 137 de 2010, tenían un pesos porcentual del $100 \%$ sobre la evaluación del desempeño laboral y era netamente de tipo individual, mientras que en el Acuerdo 565 de 2016, tienen un peso porcentual del $80 \% \%$ sobre el $100 \%$ de la evaluación del desempeño laboral del servidor con derechos de carrera, y combina evaluación de tipo individual y grupal.

- El plazo para la concertación de los compromisos laborales en el Acuerdo 137 de 2010, era hasta el 15 de febrero de cada año, mientras que en el Acuerdo 565 de 2016, es hasta el 28 de febrero de cada año.

- El término para efectuar la calificación anual u ordinaria en el Acuerdo 137 de 2010, era hasta el 15 de febrero de cada año, mientras que en el Acuerdo 565 de 2016, es máximo de 15 días hábiles siguientes al vencimiento del periodo (Téngase en cuenta que el periodo vence el 31 de enero, es decir que a partir del 1 de febrero se cuentan los 15 días hábiles).

- Para acceder al nivel sobresaliente, bajo las directrices del Acuerdo 137 de 2010, se debían cumplir con unos factores que podían ser: Evaluación de la gestión por dependencias, por calidad y oportunidad, por aportes, propuestas o iniciativas adicionales, por iniciativas tendientes a acciones proactivas en las actividades que cumpla, por participación y aprovechamiento de capacitación relacionada con las actividades propias del empleo y que genere un valor agregado para la entidad o la dependencia, por participación en grupos o en actividades que requieren de disposición voluntaria y por cumplimiento de competencias comportamentales; mientras que en el Acuerdo 565 de 2016, se elimina el cumplimiento de cualquiera de esos factores.

- Finalmente, bajo los parámetros del Acuerdo 137 de 2010, no podían ser parte de la Comisión Evaluadora, los servidores en período de prueba, mientras que bajo las directrices del Acuerdo 565 de 2016, si pueden hacer parte de dicha Comisión.

\section{CONCLUSIONES}

Bajo los parámetros del nuevo sistema "Tipo de Evaluación del Desempeño Laboral" (Acuerdo 565 de 2016) expedido por la CNSC, se asigna por primera vez a los sistemas de calificación, un valor cuantitativo a la evaluación de gestión por áreas o dependencias 
de la entidad del $10 \%$, sobre un total del $100 \%$ que en términos claros, se refiere, a la calificación del trabajo en equipo.

El trabajo en equipo se traduce en la evaluación de gestión por áreas o dependencias de la entidad que lleva a cabo el jefe de control interno y que busca crear sinergias entre cada uno de los servidores públicos y contratistas que hacen parte de esa área con la finalidad de obtener resultados más positivos en la consecución de las metas institucionales, planes de desarrollo y planes operativos anuales de la entidad.

En el nuevo sistema "Tipo de Evaluación del Desempeño Laboral" (Acuerdo 565 de 2016) expedido por la CNSC, se asigna un valor cuantitativo por primera vez, a la evaluación de las competencias comportamentales individuales del $10 \%$, sobre el $100 \%$ de la calificación total del proceso de evaluación del desempeño laboral y que busca que el servidor público con derechos de carrera posea y demuestre habilidades, actitudes y aptitudes en el desempeño de sus funciones.

Los compromisos laborales que bajo las directrices del Acuerdo 137 de 2010, equivalían a un $100 \%$ en la calificación de la evaluación del desempeño laboral de tipo netamente individual; con el nuevo Acuerdo 565 de 2016, equivalen a un $80 \%$ sobre un $100 \%$, ya que el otro $20 \%$, corresponde a un $10 \%$ de la Evaluación de Gestión por áreas o dependencias y a un $10 \%$ de las competencias comportamentales.

Los motivos que conllevaron a que la CNSC derogara el Acuerdo 137 de 2010, y que por consiguiente, expidiera el nuevo sistema "Tipo de Evaluación del Desempeño Laboral" (Acuerdo 565 de 2016), obedece a que un servidor público íntegro no sólo debe cumplir en la consecución de unos compromisos laborales de tipo individual, sino también, debe demostrar el cumplimiento de unas competencias comportamentales de carácter individual y al trabajo en equipo que se califica por el jefe de control interno de la entidad a través de la evaluación de gestión por áreas o dependencias, insumos mediante los cuales aporta a la consecución del mejoramiento institucional y que se va a reflejar en que el país sea más competitivo a nivel internacional y con una mejor percepción por parte de la ciudadanía en general.

\section{REFERENCIAS BIBLIOGRÁFICAS}

Acuerdo 565. (2016). Por el cual se establece el sistema tipo de evaluación del desempeño laboral de los empleados públicos de carrera administrativa y en periodo de prueba. Bogotá. CNSC.

Bogotá, A. (2015). Evaluación de la gestión por dependencias. Recuperado de: http://intranet. idiger.gov.co/documents/11628/20190/SEC-PT-12++Evaluaci\%C3\%B3n+de+la+gesti\% C3\%B3n+por+dependencias.pdf/e1ba6385-4944-42ab-8128-8a5eb31b9540 
Castillo, C. (2010). Modelo de gestión del talento humano por competencias. Bogotá: EditorialEscuela Superior de Administración Pública - ESAP.

Capuano. A. (2004). Evaluación de desempeño: desempeño por competencias. Argentina:Universidad del Centro Educativo Latinoamericano, 7 (13), 139-150.

Corte Constitucional (2005). C-501. Principio de estabilidad laboral en carrera administrativa. Bogotá.

Garcia, D. (2014). Competencias Comportamentales y funcionales. Recuperado de https://prezi.com/7eh3kyxl281r/competencias-comportamentales-y-funcionales/

Gil, A. (2012). Evaluación del desempeño laboral. Bogotá: Editorial Escuela Superior de Administración Pública - ESAP.

Rodríguez, J. (2013). El sistema de evaluación del desempeño laboral en Colombia: un caso de alta formalización con baja institucionalización. Bogotá. Editorial Administración y Desarrollo, 42 (58), 20-21.

Video: Se entrega en físico CD que contiene la entrevista efectuada el 23 de marzo de 2017 a la Directora de Administración de la Carrera Administrativa de la Comisión Nacional del Servicio Civil - CNSC- Dra. Sonia Patricia Cruz Ortega. 


\section{TRATAMIENTO JURÍDICO DE LAS ENERGIAS RENOVABLES EN COLOMBIA: AHORRO ENERGÉTICO, EFICIENCIA ENERGÉTICA Y USO RACIONAL DE LA ENERGÍA ${ }^{1}$}

Legal Treatment of Renewable Energies in Colombia: Energy Saving, Energy Efficiency and Rational Use of Energy

\section{$\underline{\text { Milton José Pereira Blanco }}^{2}$}

Fecha de recepción: 4 de noviembre de 2016

Fecha de aceptación: 13 de diciembre de 2016

SUMARIO: 1. Introducción; 2. Energías renovables: fundamento normativo; 3. Crisis energética y posibles salidas; 4 . Ahorro energético, uso racional y eficiente de la energía: concepto y régimen jurídico; 5. Los biocombustibles como estrategia de promoción del uso racional y eficiente de la energía; 6. Conclusiones; 7. Referencias bibliográficas.

\footnotetext{
1 El Presente trabajo constituye un avance del marco teórico de la investigación concluida, titulada Relación entre Energía, Medio Ambiente y Desarrollo Económico a partir del Análisis Jurídico de las Energías Renovables en Colombia del cual el autor es investigador principal, y fue dirigida por el Doctor en Derecho Carlos Javier Velásquez Muñoz. Este trabajo se encuentra adscrito a la línea de investigación denominada Asuntos públicos y administración de justicia del Grupo de Investigación en Derecho y Ciencia Política de la Universidad del Norte.

2 Profesor de Derecho Administrativo General, Teoría General del Derecho y Filosofía del Derecho I de la Universidad de Cartagena y de la Fundación Universitaria Colombo Internacional. Abogado y Licenciado en Filosofía (C). Magíster en Derecho, Universidad del Norte. Especialista en Derecho Contencioso Administrativo de la Universidad Externado de Colombia, y estudiante de la especialización en Derecho del Medio Ambiente de la misma Universidad. Correo electrónico: mpereira@unicolombo.edu.co y miltonjosepereirablanco@gmail.com.
} 


\section{COMO CITAR ESTE ARTÍCULO (APA 6)}

Pereira Blanco, Milton José (2017). Tratamiento jurídico de las energías renovables en Colombia: ahorro energético, eficiencia energética y uso racional de la energía. Revista Jurídica Mario Alario D’Filippo, IX (17), pág. 43-68.

\section{RESUMEN}

En este trabajo se sostiene como tesis central, que el Estado Colombiano, sí bien ha desarrollado timidamente el tema de los biocombustibles como energías renovables, la propuesta frente a otras fuentes no convencionales ha sido deficiente, porque no ha estructurado herramientas eficaces para entregar competitividad dentro del mercado energético a las energías renovables, siendo pasivo el Estado pasivo frente a la protección ambiental en cuanto a este punto se refiere. Lo anterior a partir del siguiente problema: ¿Cual ha sido el tratamiento jurídico de las energías renovables en Colombia y cual ha sido el nivel de incidencia de estas, en la protección ambiental? La tesis expuesta implica sostener que la eficiencia energética se convierte en el elemento principal de las energías renovables, dado que a partir de este tipo de energía se protege el medio ambiente. De igual forma, se debe señalar que el sistema jurídico energético colombiano no incluye en la canasta energética a las energías renovables como componente central, por cuanto la normatividad nacional solo se limita a fomentar las energías renovables sin implementar reformas estructurales al sistema en aras de hacer de las energías renovables el elemento central de la economía colombiana.

\section{PALABRAS CLAVES}

Eficiencia energética, uso racional de la energía, ahorro energético, crisis energética

\section{ABSTRACT}

This paper argues that the Colombian State has timidly developed the topic of biofuels as renewable energies. The proposal against other non-conventional sources has been deficient because it has not structured effective tools to deliver competitiveness within Of the energy market to renewable energies, the passive State being passive in relation to environmental protection in this regard. The above from the following problem: What has been the legal treatment of renewable energies in Colombia and what has been the incidence of these in environmental protection? The thesis presented implies that energy efficiency becomes the main element of renewable energies, given that this type of energy protects the environment. Likewise, it should be noted that the Colombian energy legal system does not include renewable energies in the energy basket as a central component, since national regulations are limited to promoting renewable energies without implementing structural reforms to the system for the sake of renewable energies the central element of the Colombian economy.

\section{KEYWORDS}

Energy efficiency, rational use of energy, energy saving, energy crisis 